\title{
5. SUMMARY OF GEOPHYSICAL DATA FROM THE SULU AND CELEBES SEAS1
}

\author{
K. Hinz ${ }^{2}$ and M. Block ${ }^{2}$
}

\section{INTRODUCTION}

A diversity of geophysical data exist from the Sulu and $\mathrm{Ce}$ lebes seas. Two seismic refraction profiles and magnetic surveyss were made by the Vema in 1967 (Murauchi et al., 1973). In 1974 the Comité d'Etudes Petroliers Marines conducted a multichannel seismic survey across the Sulu Sea (Mascle and Biscarrat, 1978). The Federal Institute for Geosciences and Natural Resources (BGR) collected 10,315 km of multichannel seismic data in parallel with magnetic and gravimetric measurements in the Sulu Sea and $580 \mathrm{~km}$ in the Celebes Sea on six cruises during the period from 1977 to 1987 (Table 1; Hinz et al., 1986; Dürbaum and Hinz, 1983). The oil industry surveyed approximately $18,000 \mathrm{~km}$ of multichannel seismic (MCS) data on the western Sulu shelf from 1965 to 1974 (Bell and Jessop, 1974).

Aeromagnetic measurements were carried out over the northwest Sulu Sea shelf (Bosum et al., 1972), and the U.S. Naval Oceanographic Office conducted a Project Magnet aeromagnetic survey of the central region of the Sulu Sea in 1987.

Heat-flow measurements (Tables 2 and 3) and sonobuoy seismic refraction measurements (Tables 4 and 5) were made in the Sulu Sea.

\section{STRUCTURAL UNITS OF THE SOUTHEASTERN SULU BASIN}

The main structural features observed in the Sulu Sea are shown in Figure 1. We identified five structural zones within the southeastern Sulu Basin on the basis of seismic interpretations. Some of these lines are shown in Figure 2 (back pocket).

\section{Zone SES-I}

Zone SES-I comprises the major portion of the deep southeastern Sulu basin. It lies between deep bathymetric depressions off the Sulu Archipelago and off Mindanao and Negros islands, and the steep southeast-facing flank of the Cagayan Ridge. A distinct $\mathrm{N} 25^{\circ} \mathrm{E}$-trending shear zone forms its western boundary. Zone SES-I is $130-140 \mathrm{~km}$ wide, but narrows east of $121.5^{\circ} \mathrm{E}$ toward the northeast. Its igneous basement is relatively smooth, and it has seismic characteristics typical of oceanic crust overlain by a 1-2-s (twt) thick sedimentary unit.

The dominant structural feature of Zone SES-I is a N $60^{\circ} \mathrm{E}$ trending chain of basement highs. The largest observed basement high is $60 \mathrm{~km}$ long, $15 \mathrm{~km}$ wide, and approximately 1500 $\mathrm{m}$ high (Fig. 1). Basaltic rocks have been dredged from its eastern flank (Kudrass et al., this volume). The basement deepens from the chain of basement highs toward the southeast (i.e., toward the Sulu Archipelago and the islands of Mindanao, respectively; Fig. 1).

The heat-flow values are relatively high (Table 2). A distinct heat-flow anomaly with values ranging from 110 to $198 \mathrm{~mW} / \mathrm{m}^{2}$

\footnotetext{
${ }^{1}$ Rangin, C., Silver, E., von Breymann, M. T., et al., 1990. Proc. ODP, Init. Repts., 124: College Station, TX (Ocean Drilling Program).

2 Bundesanstalt für Geowissenschaften und Rohstoffe, D3000 Hannover 51, Postfach 510153, Federal Republic of Germany.
}

coincides roughly with the eastern and southeastern boundary of Zone SES-I. Neither our own shipborne magnetic data nor the Project Magnet aeromagnetic data confirm the existence of northeast-trending magnetic lineations within Zone SES-I, described and interpreted by Lee and McCabe (1986) as magnetic lineations 17-20 (41-47 m.y.). Earthquakes are rare within Zone SES-I.

\section{Zone SES-II}

Zone SES-II is $7-25 \mathrm{~km}$ wide and comprises the more than 4550-m-deep bathymetric depressions off Mindanao and Negros islands that are trenches of an active subduction zone. The eastward- to southeastward-descending oceanic basement with dips of $11^{\circ}$ is overlain by a $0.5-1.8-\mathrm{s}$ (twt) sedimentary section. Temperatures of $+10^{\circ} \mathrm{C}$ have been measured at the seabed, and heat-flow values ranging from 78 to $99 \mathrm{~mW} / \mathrm{m}^{2}$ have been determined within Zone SES-II. Sediments recovered by piston coring from this zone are finely laminated and multicolored, and are interpreted as turbidites (Kudrass et al., this volume). Zone SES-II terminates against Zone SES-III at about $9.5^{\circ} \mathrm{N}$.

\section{Zone SES-III}

Zone SES-III is $15-35 \mathrm{~km}$ wide and is off the Sulu Archipelago east of $120^{\circ} \mathrm{E}$, off Zamboanga Peninsula, and off Negros Island (Fig. 1). Its trend changes from $\mathrm{N} 60^{\circ} \mathrm{E}$ off the Sulu Archipelago to $\mathrm{N}-\mathrm{S}$ at $7.5^{\circ} \mathrm{N}$.

Zone SES-III is characterized by a series of imbricate thrust sheets forming a complex thrust system of predominantly sedimentary origin, as suggested from the derived normal moveout (NMO) velocities. The individual imbricate thrust sheets are separated by faults dipping to the southeast and east, respectively. The faults curve downward to a sole fault or major detachment plane that lies just above the descending igneous oceanic crust (Fig. 2 [back pocket] and Figs. 3 and 4).

The thickness of the complex thrust system comprising Zone SES-III increases landward to more than $4 \mathrm{~s}$ (twt), that is, about $6000 \mathrm{~m}$. Zone SES-III can be described as an accretionary wedge resulting from active subduction of igneous oceanic crust, as indicated by the conspicuous bench-seafloor topography at the toe of the wedge and active seismicity.

Zone SES-III collides with the structural zone CA-I of the Cagayan terrane at $9.5^{\circ} \mathrm{N}$, resulting in the formation of a distinct and complex anticlinal stack structure north of $9.5^{\circ} \mathrm{N}$ that continues northward into the Antique Range of western Panay Island. The latter is composed of a polymict melange that contains blocks of glaucophane schist, gabbro, peridotite, metavolcanics, and red chert embedded in a serpentinite matrix (McCabe et al., 1982). The Oligocene Panpanan Basalt occupies much of the northeast-trending Antique Range watershed west of the Iloilo Basin, according to Mitchell et al. (1986). North of $10^{\circ} \mathrm{N}$, Zone SES-III is juxtaposed against and even thrust onto the Cagayan Ridge (Fig. 1).

The seismic data suggest that at least the southern offshore part of the Antique Ridge represents a collisional belt consisting of the accretionary wedge of structural Zone SES-III and the in- 
Table 1. Multichannel seismic, magnetic, and gravimetric surveys in the Celebes and Sulu seas.

\begin{tabular}{|c|c|c|c|c|c|c|c|c|c|c|}
\hline \multirow[b]{2}{*}{ Year } & \multirow[b]{2}{*}{$\begin{array}{l}\text { Vessel/ } \\
\text { country }\end{array}$} & \multirow[b]{2}{*}{ Cruise } & \multicolumn{2}{|c|}{ Seismic source } & \multicolumn{2}{|c|}{ Receiver } & \multirow[b]{2}{*}{$\begin{array}{c}\text { Coverage } \\
(\%)\end{array}$} & \multirow{2}{*}{$\begin{array}{c}\text { Sonobuoy } \\
\text { station } \\
\text { no. }\end{array}$} & \multirow{2}{*}{$\begin{array}{l}\text { Gravity } \\
\text { and } \\
\text { magnetics }\end{array}$} & \multirow{2}{*}{$\begin{array}{c}\text { Total } \\
\text { survey } \\
(\mathrm{km})\end{array}$} \\
\hline & & & $\begin{array}{l}\text { Volume } \\
{\text { (in. }{ }^{3} \text { ) }}^{\text {Vol }}\end{array}$ & $\begin{array}{l}\text { No. of } \\
\text { guns }\end{array}$ & $\begin{array}{l}\text { Streamer } \\
\text { length }(\mathrm{m})\end{array}$ & $\begin{array}{c}\text { No. of } \\
\text { channels }\end{array}$ & & & & \\
\hline 1974 & $\begin{array}{l}\text { Lady Gloritta } \\
\text { France }\end{array}$ & CEPM & Vaporchoc & & 2,400 & 24 & 2,400 & & & $\sim 600$ \\
\hline 1977 & $\begin{array}{l}\text { Valdivia } \\
\text { F.R. Germany }\end{array}$ & VA-16 & 900 & 3 & 2,400 & 24 & 2,400 & 7 & $\mathrm{G}, \mathrm{M}$ & 1,955 \\
\hline 1982 & $\begin{array}{l}\text { Sonne } \\
\text { F.R. Germany }\end{array}$ & SO-23 & 1,562 & 10 & $\begin{array}{l}2,400 \\
1,200\end{array}$ & $\begin{array}{l}24 \\
12\end{array}$ & $\begin{array}{l}2,400 \\
1,200\end{array}$ & 14 & G, M & 3,300 \\
\hline 1983 & $\begin{array}{l}\text { Sonne } \\
\text { F.R. Germany }\end{array}$ & SO-27 & 1,562 & 10 & 2,400 & 24 & 2,400 & & G, M & 227 \\
\hline 1984 & $\begin{array}{l}\text { Explora } \\
\text { F.R. Germany }\end{array}$ & BGR-84 & 3,512 & 31 & 3,000 & 60 & 3,000 & & & 1,041 \\
\hline 1986 & $\begin{array}{l}\text { Explora } \\
\text { F.R. Germany }\end{array}$ & BGR-86 & 2,756 & 28 & 3,000 & 60 & 3,000 & & G, M & 1,672 \\
\hline 1987 & $\begin{array}{l}\text { Sonne } \\
\text { F.R. Germany }\end{array}$ & SO-49 & 1,562 & 10 & 2,400 & 48 & 2,400 & & G, M & 2,120 \\
\hline \multicolumn{11}{|c|}{ Celebes Sea } \\
\hline \multirow[t]{2}{*}{1987} & $\begin{array}{l}\text { Sonne } \\
\text { Germany }\end{array}$ & SO-49 & 1,562 & 10 & 2,400 & 48 & 2,400 & & G, M & 580 \\
\hline & & & & & & & & & $\Sigma$ & 11,495 \\
\hline
\end{tabular}

ferred volcanic Zone CA-I. North of $10^{\circ} \mathrm{N}$, this complex collisional belt is juxtaposed and even thrust onto the Cagayan Ridge (Fig. 1).

\section{Zone SES-IV}

Zone SES-IV comprises an area of a particularly pronounced structural style. The $15-30-\mathrm{km}$ wide zone extends over $300 \mathrm{~km}$ from the continental margin of southern Panay in the north, toward $7^{\circ} 30^{\prime} \mathrm{S}$ in the south (Fig. 1). Because of the lack of MCS data, it is uncertain whether this zone exists beneath the northern frontal portion of the Sulu Archipelago.

Zone SES-IV is characterized by three superimposed tectonic-stratigraphic units overlying actively subducting oceanic basement. The dominant tectonic-stratigraphic unit is a regional splinter characterized by high-amplitude reflections at the top and the base, and interval velocities of $>4.5 \mathrm{~km} / \mathrm{s}$. The thickness of this regional splinter increases from less than $0.5 \mathrm{~s}$ (twt) at the front to $2.2 \mathrm{~s}$ (twt) toward the central Philippine Islands. Our seismic data strongly suggest that the splinter forms the "backstop" against which the previously discussed Zone SESIII accretes by the development of imbricate thrust sheets and simultaneous duplex-type shortening within the zone. By this process, mass is added to Zone SES-III resulting in thickening and progressive seaward growth of the zone as well as simultaneous uplift of the splinter.

The inferred oceanic crust slab is overlain by a sedimentary apron up to $2.4 \mathrm{~s}$ (twt) thick. The individual sedimentary sequence boundaries of this apron have a gentle dip of about $8^{\circ}$ toward the east and downlap onto the top of the splinter (Fig. 2 ). Above the inferred hinge zone between the oceanic crustal splinter and the Philippine island arc (i.e. Zone SES-V) the apron sediments are folded and thrusted, resulting in the buildup of a complex anticlinal structure (Figs. 3 and 4). North of $9.5^{\circ} \mathrm{N}$ the sedimentary apron and also the underlying inferred oceanic crustal slab are affected by collision-related backthrusting. Apparently the sedimentary apron continues northward into the Iloilo basin of central Panay, which contains limestones, coarse clastic sediments, and interbedded basalt flows of Oligocene-lower Miocene age, overlain by a middle Miocene to Pliocene sequence of fine-grained sandstone and shale (McCabe et al., 1982).
Heat-flow values ranging from 45 to $70 \mathrm{~mW} / \mathrm{m}^{2}$ have been determined within Zone SES-IV, and the depths of the seismic epicenters are less than $75 \mathrm{~km}$.

\section{Zone SES-V}

Zone SES-V comprises the western slope and shelf of Negros Island and of the Zamboanga Peninsula. A sedimentary apron with variable thickness ranging from a few hundred meters to $2.8 \mathrm{~s}$ (twt) overlies an acoustic basement complex, the surface of which is often characterized by a high-amplitude, discontinuous reflection horizon (Figs. 3 and 4).

The distinct acoustic basement complex of Zone SES-V presumably represents subsided parts of the Cretaceous melange basement, including overlying Oligocene to Miocene volcanic rocks of the Zamboanga Peninsula and of Negros Island. The melange basement of Zamboanga Peninsula includes chlorite and hornblende schists, metasandstone, phyllite, slate, marble, Cretaceous limestone, volcanic rocks, and lenses of serpentinized peridotite (Hamilton, 1979). Cretaceous sandstone, shale, spilite, and pillow basalt crop out in the southwest part of $\mathrm{Ne}$ gros Island, which otherwise consists mostly of Neogene andesitic volcanic rocks and their intrusive equivalents and derivative volcaniclastic sedimentary rocks (Hamilton, 1979).

\section{REFERENCES}

Bell, R. M., and Jessop, R.G.C., 1974. Exploration and geology of the west Sulu Basin, Philippines. APEA J., 14:21-28.

Block, M., and Steinmann, D., 1988a. Geothermal studies. In Kudrass, H. R., et al. (Eds.), Geological, Geochemical and Geothermal Investigations in the Sulu Sea/Phillipines (SO 49). BGR Rep. 102042, Pt. 3 (Hannover, F. R. Germany).

Block, M., and Steinmann, D., 1988b. Geothermal studies. In Kudrass, H. R., et al. (Eds.), Geological, Geochemical and Geothermal Investigations in the Sulu Sea/Phillipines (SO 58). BGR Rep. 104774, Pt. 2 (Hannover, F. R. Germany).

Bosum, W., Fernandez, E., Kind, E., and Theodoro, C., 1972. Aeromagnetic survey of the Palawan Sulu offshore of the Philippines. CCOP Tech. Bull., 6:141-160.

Dürbaum, H. J., and Hinz, K., 1983. SEATAR-related geophysical studies of the BGR in the Southwest Pacific. Transactions, CircumPacific Energy and Mineral Resources Conference, 3rd, August 1982, Honolulu, HI, 129-133. 
Hamilton, W., 1979. Tectonics of the Indonesian region. U.S. Geol. Surv. Prof. Pap., 1078.

Hinz, K., Kempter, E.H.K., Schlüter, H. U., 1986. The southern Palawan Balabac area: an accreted or non-accreted terrane? Proc. Asian Council on Petroleum (ASCOPE) Conference and Exhibition, 3rd, Dec. 1985, Kuala Lumpur, Malaysia, 2:48-72.

Hinz, K., and Schlüter, H. U., 1985. Geology of Dangerous Grounds, South China Sea, and the continental margin off Southwest Palawan-results of Sonne cruises, SO23 and SO27. Energy, 10:297315.

Lee, C. S., and McCabe, R., 1986. The Banda-Celebes Sulu Basin: a trapped piece of Cretaceous-Eocene oceanic crust. Nature, 322:5154.

Ludwig, W. J., Kumar, N., and Houtz, R. E., 1979. Profiler-sonobuoy measurements in the South China Sea basin. J. Geophys. Res., 84: 3505-3518.

McCabe, P. R., Almasco, J., and Diegor, W., 1982. Geologic and paleomagnetic evidence for a possible Miocene collision in western Panay, central Philippines. Geology, 10:325-329.
Mascle, A., and Biscarrat, P. A., 1978. The Sulu Sea: a marginal basin in Southeast Asia. In Watkins, J. S., Montadert, L., and Dickerson, P. W., (Eds.), Geological and Geophysical Investigation of Continental Margins. AAPG Mem., 29:373-381.

Mitchell, A.H.G., Hernandez, F., and De la Cruz, A. P., 1986. Cenozoic evolution of the Philippine Archipelago. J. Southeast Asian Earth Sci., 1:3-22.

Murauchi, S., Ludwig, W. J., Den, N., Hotta, H., Asanuma, T., Yoshii, T., Kubotera, A., and Hagiwara, K., 1973. Structure of the Sulu Sea and Celebes Sea. J. Geophys. Res., 78:3437-3447.

Nagasaka, K., Francheteau, J., and Kishii, T., 1970. Terrestrial heat flow in the Celebes and Sulu seas. Mar. Geophys. Res., 1:99-103.

Sclater, J. G., Karig, D., Lawver, L. A., and Louden, K., 1976. Heat flow, depth, and crustal thickness of the marginal basins of the South Philippine Sea. J. Geophys. Res., 81:309-318.

Ms 124A-105

Table 2. Geothermal measurements in the southeast Sulu Sea Basin.

\begin{tabular}{|c|c|c|c|c|c|c|c|c|c|}
\hline \multirow{2}{*}{$\begin{array}{l}\text { Number } \\
\text { source }\end{array}$} & \multirow{2}{*}{$\begin{array}{l}\text { Name of } \\
\text { station }\end{array}$} & \multicolumn{2}{|c|}{ Location } & \multirow{2}{*}{$\begin{array}{c}\text { Water } \\
\text { depth } \\
(\mathrm{m})\end{array}$} & \multirow{2}{*}{$\begin{array}{c}\text { Gradient } \\
\left({ }^{\circ} \mathrm{C} / \mathrm{cm} \times 10^{3}\right)\end{array}$} & \multicolumn{2}{|c|}{$\begin{array}{l}\text { Conductivity } \\
\left(\mathrm{cal} / \mathrm{cm} \cdot{ }^{-5}\right. \\
\left.{ }^{\circ} \mathrm{C} \times 10^{3}\right)\end{array}$} & \multicolumn{2}{|c|}{ Heat Flow } \\
\hline & & $\mathrm{N}$ & E & & & in situ & core & $\left(\mu \mathrm{cal} / \mathrm{cm}^{2} \cdot \mathrm{s}\right)$ & $\left(\mathrm{mW} / \mathrm{m}^{2}\right)$ \\
\hline $1 / \mathrm{A}$ & Circle 15 & $7^{\circ} 40.0^{\prime}$ & $121^{\circ} 28.0^{\prime}$ & 4974 & 1.06 & \multicolumn{2}{|c|}{2.1 (assumed) } & 2.2 & 92 \\
\hline 2/A & Circle 16 & $8^{\circ} 21.1^{\prime}$ & $120^{\circ} 56.4^{\prime}$ & 4295 & 1.07 & \multicolumn{2}{|c|}{2.1 (assumed) } & 2.3 & 96 \\
\hline $3 / \mathrm{A}$ & Circle 17 & $7^{\circ} 43.4^{\prime}$ & $119^{\circ} 34.8^{\prime}$ & 3675 & 1.23 & \multicolumn{2}{|c|}{2.1 (assumed) } & 2.6 & 108 \\
\hline 4/B & A 181 & $7^{\circ} 17.6^{\prime}$ & $119^{\circ} 45.9^{\prime}$ & 3801 & 1.08 & \multicolumn{2}{|c|}{1.74 (assumed) } & 1.90 & 79 \\
\hline $5 / \mathrm{B}$ & A 182 & $6^{\circ} 41.0^{\prime}$ & $120^{\circ} 09.0^{\prime}$ & 3641 & 1.02 & - & 1.74 & 1.77 & 74 \\
\hline $6 / B$ & T 01 & $8^{\circ} 17.5^{\prime}$ & $120^{\circ} 50.2^{\prime}$ & 3932 & 1.03 & \multicolumn{2}{|c|}{1.90 (assumed) } & 1.96 & 82 \\
\hline $7 / \mathrm{B}$ & T 02 & $8^{\circ} 25.8^{\prime}$ & $121^{\circ} 43.1^{\prime}$ & 4857 & 0.31 & \multicolumn{2}{|c|}{1.90 (assumed) } & 0.59 & 25 \\
\hline $8 / \mathrm{C}$ & HF $67 / 1$ & $9^{\circ} 26.622^{\prime}$ & $121^{\circ} 54.619^{\prime}$ & 4023 & 0.48 & 2.23 & 2.05 & 1.07 & 45 \\
\hline 9/C & HF $67 / 2$ & $9^{\circ} 26.493^{\prime}$ & $121^{\circ} 54.628^{\prime}$ & 4017 & 0.49 & 2.13 & 2.05 & 1.04 & 43 \\
\hline $10 / C$ & HF 69 & $9^{\circ} 21.406^{\prime}$ & $121^{\circ} 38.678^{\prime}$ & 4635 & 1.09 & 2.17 & 2.10 & 2.37 & 99 \\
\hline $11 / \mathrm{C}$ & HF $76 / 1$ & $9^{\circ} 16.687^{\prime}$ & $121^{\circ} 32.067^{\prime}$ & 4642 & 1.91 & 2.30 & 2.18 & 4.39 & 183 \\
\hline $12 / \mathrm{C}$ & HF $76 / 2$ & $9^{\circ} 16.611^{\prime}$ & $121^{\circ} 32.103^{\prime}$ & 4650 & 2.09 & 2.27 & 2.18 & 4.74 & 198 \\
\hline $13 / C$ & HF $81 / 1$ & $8^{\circ} 09.808^{\prime}$ & $121^{\circ} 36.383^{\prime}$ & 4804 & 1.60 & 2.15 & 2.14 & 3.44 & 143 \\
\hline $14 / \mathrm{C}$ & HF $81 / 2$ & $8^{\circ} 09.902^{\prime}$ & $121^{\circ} 36.336^{\prime}$ & 4835 & 1.48 & 2.17 & 2.14 & 3.21 & 134 \\
\hline $15 / \mathrm{C}$ & HF 92 & $8^{\circ} 01.43^{\prime}$ & $121^{\circ} 56.96^{\prime}$ & 3986 & 0.56 & 2.84 & 2.43 & 1.59 & 66 \\
\hline $16 / C$ & HF 97 & $8^{\circ} 11.00^{\prime}$ & $119^{\circ} 28.32^{\prime}$ & 3564 & 1.07 & 1.97 & 1.91 & 2.11 & 88 \\
\hline $17 / \mathrm{D}$ & $41 \mathrm{HF}-1$ & $9^{\circ} 56.09^{\prime}$ & $121^{\circ} 39.09^{\prime}$ & 3758 & 0.40 & 2.21 & - & 0.88 & 37 \\
\hline $18 / \mathrm{D}$ & $41 \mathrm{HF}-2$ & $9^{\circ} 56.51^{\prime}$ & $121^{\circ} 39.57^{\prime}$ & 3755 & 0.34 & 2.15 & - & 0.73 & 30 \\
\hline 19/D & SOHF-1 & $9^{\circ} 16.35^{\prime}$ & $121^{\circ} 29.84^{\prime}$ & 4379 & 1.66 & \multicolumn{2}{|c|}{2.18 (assumed) } & 3.62 & 151 \\
\hline 20/D & $50 \mathrm{HF}-2$ & $9^{\circ} 16.46^{\prime}$ & $121^{\circ} 30.12^{\prime}$ & 4398 & 1.79 & 2.18 & - & 3.90 & 163 \\
\hline $21 / \mathrm{D}$ & $50 \mathrm{HF}-3$ & $9^{\circ} 16.34^{\prime}$ & $121^{\circ} 30.41^{\prime}$ & 4420 & 2.11 & \multicolumn{2}{|c|}{2.18 (assumed) } & 4.60 & 192 \\
\hline $22 / \mathrm{D}$ & $50 \mathrm{HF}-4$ & $9^{\circ} 16.37^{\prime}$ & $121^{\circ} 30.87^{\prime}$ & 4494 & 1.39 & 2.27 & - & 3.16 & 131 \\
\hline $23 / \mathrm{D}$ & 55HF-1 & $9^{\circ} 23.42^{\prime}$ & $121^{\circ} 40.14^{\prime}$ & 4567 & 0.98 & 2.22 & - & 2.18 & 91 \\
\hline 24/D & 55HF-2 & $9^{\circ} 22.02^{\prime}$ & $121^{\circ} 39.83^{\prime}$ & 4611 & 0.99 & 2.51 & - & 2.48 & 104 \\
\hline $25 / \mathrm{D}$ & $55 \mathrm{HF}-3$ & $9^{\circ} 22.64^{\prime}$ & $121^{\circ} 39.54^{\prime}$ & 4611 & 0.92 & 2.40 & - & 2.21 & 92 \\
\hline $26 / \mathrm{D}$ & $55 \mathrm{HF}-4$ & $9^{\circ} 22.66^{\prime}$ & $121^{\circ} 39.00^{\prime}$ & 4585 & 1.09 & 2.19 & - & 2.39 & 99 \\
\hline $27 / D$ & 55HF-5 & $9^{\circ} 22.37^{\prime}$ & $121^{\circ} 38.47^{\prime}$ & 4570 & 1.04 & 2.17 & - & 2.26 & 94 \\
\hline $28 / \mathrm{D}$ & $55 \mathrm{HF}-6$ & $9^{\circ} 22.09^{\prime}$ & $121^{\circ} 37.97^{\prime}$ & 4659 & 1.16 & 2.11 & - & 2.45 & 102 \\
\hline 29/D & $55 \mathrm{HF}-7$ & $9^{\circ} 21.70^{\prime}$ & $121^{\circ} 37.43^{\prime}$ & 4696 & 1.11 & \multicolumn{2}{|c|}{2.15 (assumed) } & 2.39 & 99 \\
\hline $30 / \mathrm{D}$ & $60 \mathrm{HF}$ & $9^{\circ} 14.10^{\prime}$ & $121^{\circ} 25.40^{\prime}$ & 4063 & 0.38 & 2.14 & - & 0.81 & 34 \\
\hline $31 / \mathrm{D}$ & $61 \mathrm{HF}$ & $9^{\circ} 09.2^{\prime}$ & $121^{\circ} 17.1^{\prime}$ & 3693 & 0.76 & 2.04 & - & 1.55 & 65 \\
\hline $32 / \mathrm{D}$ & $62 \mathrm{HF}$ & $9^{\circ} 05.26^{\prime}$ & $121^{\circ} 11.44^{\prime}$ & 3920 & 1.03 & \multicolumn{2}{|c|}{2.15 (assumed) } & 2.21 & 92 \\
\hline $33 / \mathrm{D}$ & $68 \mathrm{HF}$ & $8^{\circ} 49.80^{\prime}$ & $121^{\circ} 20.00^{\prime}$ & 3350 & 0.40 & 2.01 & - & 0.80 & 34 \\
\hline $34 / D$ & $70 \mathrm{HF}$ & $8^{\circ} 49.80^{\prime}$ & $121^{\circ} 35.40^{\prime}$ & 4726 & 1.71 & 2.18 & - & 3.73 & 155 \\
\hline $35 / \mathrm{D}$ & $75 \mathrm{HF}$ & $9^{\circ} 21.19^{\prime}$ & $121^{\circ} 35.74^{\prime}$ & 4700 & 1.09 & \multicolumn{2}{|c|}{2.15 (assumed) } & 2.34 & 98 \\
\hline $36 / \mathrm{D}$ & 79 HF-1 & $8^{\circ} 24.50^{\prime}$ & $121^{\circ} 10.46^{\prime}$ & 4270 & 1.09 & 2.05 & - & 2.23 & 93 \\
\hline $37 / D$ & $79 \mathrm{HF}-2$ & $8^{\circ} 24.70^{\prime}$ & $121^{\circ} 10.70^{\prime}$ & 4270 & 1.08 & 2.03 & - & 2.19 & 91 \\
\hline $38 / \mathrm{D}$ & $81 \mathrm{HF}$ & $7^{\circ} 59.70^{\prime}$ & $121^{\circ} 15.00^{\prime}$ & 4320 & 1.29 & 2.05 & - & 2.64 & 110 \\
\hline 39/D & $84 \mathrm{HF}$ & $8^{\circ} 08.60^{\prime}$ & $121^{\circ} 39.00^{\prime}$ & 4870 & 0.90 & 2.09 & - & 1.87 & 78 \\
\hline $40 / \mathrm{D}$ & $85 \mathrm{HF}$ & $8^{\circ} 09.03^{\prime}$ & $121^{\circ} 41.49^{\prime}$ & 4870 & 0.98 & 2.08 & - & 2.04 & 85 \\
\hline $41 / \mathrm{D}$ & $87 \mathrm{HF}$ & $8^{\circ} 02.50^{\prime}$ & $121^{\circ} 53.90^{\prime}$ & 3906 & 0.58 & 2.26 & - & 1.31 & 55 \\
\hline $42 / \mathrm{D}$ & $91 \mathrm{HF}$ & $8^{\circ} 15.60^{\prime}$ & $121^{\circ} 30.57^{\prime}$ & 4460 & 1.11 & 2.12 & - & 2.35 & 98 \\
\hline 43/D & $92 \mathrm{HF}$ & $8^{\circ} 12.75^{\prime}$ & $121^{\circ} 34.15^{\prime}$ & 4560 & 1.28 & 2.11 & - & 2.70 & 113 \\
\hline 44/D & $99 \mathrm{HF}$ & $8^{\circ} 09.00^{\prime}$ & $121^{\circ} 44.50^{\prime}$ & 4680 & 0.97 & 2.00 & - & 1.94 & 81 \\
\hline 45/D & $100 \mathrm{HF}$ & $8^{\circ} 08.77^{\prime}$ & $121^{\circ} 47.40^{\prime}$ & 4498 & 0.78 & 1.95 & - & 1.52 & 63 \\
\hline $46 / \mathrm{D}$ & $103 \mathrm{HF}$ & $8^{\circ} 09.24^{\prime}$ & $121^{\circ} 38.70^{\prime}$ & 4870 & 1.38 & 2.11 & - & 2.91 & 121 \\
\hline
\end{tabular}

Sources: $\mathrm{A}=$ Nagasaka et al., 1970; $\mathrm{B}=$ Sclater et al., 1976; C = Sonne cruise SO-49, Block and Steinmann, 1988a; and D = Sonne cruise SO-58, Block and Steinmann, 1988 b. 
Table 3. Geothermal measurements in the northwest Sulu Sea Basin.

\begin{tabular}{|c|c|c|c|c|c|c|c|c|c|}
\hline \multirow{2}{*}{$\begin{array}{l}\text { Number } \\
\text { source }\end{array}$} & \multirow{2}{*}{$\begin{array}{l}\text { Name of } \\
\text { station }\end{array}$} & \multicolumn{2}{|c|}{ Location } & \multirow{2}{*}{$\begin{array}{l}\text { Water } \\
\text { depth } \\
\text { (m) }\end{array}$} & \multirow{2}{*}{$\begin{array}{c}\text { Gradient } \\
\left({ }^{\circ} \mathrm{C} / \mathrm{cm} \times 10^{3}\right)\end{array}$} & \multicolumn{2}{|c|}{$\begin{array}{l}\text { Conductivity } \\
(\mathrm{cal} / \mathrm{cm} \cdot \mathrm{s} \\
\left.\cdot{ }^{\circ} \mathrm{C} \times 10^{3}\right)\end{array}$} & \multicolumn{2}{|c|}{ Heat Flow } \\
\hline & & $\mathrm{N}$ & E & & & in situ & core & $\left(\mu \mathrm{cal} / \mathrm{cm}^{2} \cdot \mathrm{s}\right)$ & $\left(\mathrm{mW} / \mathrm{m}^{2}\right)$ \\
\hline $47 / C$ & HF $46 / 1$ & $10^{\circ} 01.395^{\prime}$ & $120^{\circ} 29.474^{\prime}$ & 1542 & 0.71 & 1.94 & 2.14 & 1.38 & 58 \\
\hline $48 / C$ & HF $46 / 2$ & $10^{\circ} 01.352^{\prime}$ & $120^{\circ} 29.637^{\prime}$ & 1542 & 0.74 & 2.10 & 2.14 & 1.55 & 65 \\
\hline $49 / C$ & HF $51 / 1$ & $10^{\circ} 21.308^{\prime}$ & $121^{\circ} 20.763^{\prime}$ & 1167 & 0.82 & 2.06 & 1.95 & 1.69 & 70 \\
\hline $50 / \mathrm{C}$ & HF $51 / 2$ & $10^{\circ} 21.077^{\prime}$ & $121^{\circ} 20.363^{\prime}$ & 1170 & 0.80 & 2.08 & 1.95 & 1.66 & 69 \\
\hline $51 / \mathrm{C}$ & HF 114 & $8^{\circ} 04.411^{\prime}$ & $118^{\circ} 19.156^{\prime}$ & 461 & 0.50 & 2.32 & 2.06 & 1.16 & 48 \\
\hline
\end{tabular}

Source: $\mathrm{C}=$ Sonne cruise SO-49, Block and Steinmann, 1988a.

Table 4. Refraction seismic and sonobuoy measurements in the northwest Sulu Sea.

\begin{tabular}{|c|c|c|c|c|c|c|c|c|c|c|c|c|}
\hline \multirow{2}{*}{$\begin{array}{l}\text { Name } \\
\text { of } \\
\text { station }\end{array}$} & \multirow[b]{2}{*}{ Source } & \multicolumn{2}{|c|}{ Location } & \multirow{2}{*}{$\begin{array}{l}\text { Water } \\
\text { depth } \\
\text { (m) }\end{array}$} & \multirow{2}{*}{$\begin{array}{l}\mathrm{V}_{1} \\
\mathrm{~h}_{1}\end{array}$} & \multirow{2}{*}{$\begin{array}{l}V_{2} \\
h_{2}\end{array}$} & \multirow{2}{*}{$\begin{array}{l}V_{3} \\
h_{3}\end{array}$} & \multirow{2}{*}{$\begin{array}{l}v_{4} \\
h_{4}\end{array}$} & \multirow{2}{*}{$\begin{array}{l}V_{5} \\
h_{5}\end{array}$} & \multirow{2}{*}{$\begin{array}{l}V_{6} \\
h_{6}\end{array}$} & \multirow{2}{*}{$\begin{array}{l}V_{7} \\
h_{7}\end{array}$} & \multirow{2}{*}{$\begin{array}{l}V_{8} \\
h_{8}\end{array}$} \\
\hline & & $\mathrm{N}$ & E & & & & & & & & & \\
\hline \multirow{3}{*}{ Profile 15} & & & & & $1.90^{*}$ & 3.10 & $4.82^{\circ}$ & 5.65 & 6.72 & & & \\
\hline & A & $09^{\circ} 29^{\prime}$ & $119^{\circ} 09^{\prime}$ & 2030 & 3.00 & 4.58 & 1.29 & 3.77 & & & & \\
\hline & & $08^{\circ} 51^{\prime}$ & $119^{\circ} 15^{\prime}$ & 2030 & 1.10 & 0.70 & 4.16 & 3.79 & & & & \\
\hline \multirow{3}{*}{$307 \mathrm{C} 12$} & & & & & 2.20 & 2.95 & 3.45 & 3.85 & & & & \\
\hline & B & $07^{\circ} 15.2^{\prime}$ & $118^{\circ} 05.6^{\prime}$ & 90 & 1.18 & 1.03 & 1.35 & & & & & \\
\hline & & & & & 2.10 & 2.55 & 3.20 & 4.20 & & & & \\
\hline $308 \mathrm{Cl} 2$ & B & $06^{\circ} 50.8^{\prime}$ & $118^{\circ} 15.3^{\prime}$ & 80 & 1.13 & 0.49 & 1.54 & & & & & \\
\hline \multirow{3}{*}{$43 \mathrm{C} 14$} & & & & & 2.15 & 2.40 & 2.60 & 4.90 & & & & \\
\hline & B & $07^{\circ} 57.0^{\prime}$ & $117^{\circ} 11.5^{\prime}$ & 190 & 0.51 & 1.01 & 1.26 & & & & & \\
\hline & & & & & 1.92 & 2.24 & 3.89 & 4.25 & 4.76 & 5.86 & & \\
\hline SB-I & C & $10^{\circ} 14.49^{\prime}$ & $119^{\circ} 50.62^{\prime}$ & 110 & 0.10 & 0.48 & 0.18 & 0.94 & 1.49 & & & \\
\hline \multirow{3}{*}{ SB-II } & & & & & 1.74 & 1.97 & 2.72 & 2.95 & 3.94 & 4.5 & & \\
\hline & C & $10^{\circ} 20.98^{\prime}$ & $120^{\circ} 05.46^{\prime}$ & 190 & 0.05 & 0.28 & 0.73 & 1.38 & 0.34 & & & \\
\hline & & & & & 2.30 & 2.77 & 3.47 & 4.03 & & & & \\
\hline SB-III & C & $10^{\circ} 00.29^{\prime}$ & $120^{\circ} 36.31^{\prime}$ & 1540 & 1.59 & 0.70 & 2.06 & & & & & \\
\hline \multirow[b]{2}{*}{ SB-IV } & & & & & 2.12 & 2.55 & 3.02 & 3.54 & 4.16 & 6.66 & & \\
\hline & C & $09^{\circ} 44.02^{\prime}$ & $119^{\circ} 51.89^{\prime}$ & 1170 & 1.48 & 1.35 & 0.35 & 1.72 & 1.85 & & & \\
\hline \multirow[b]{2}{*}{ SB-V } & & & & & 1.90 & 2.21 & 2.42 & 4.21 & 4.95 & & & \\
\hline & C & $09^{\circ} 18.07^{\prime}$ & $118^{\circ} 49.92^{\prime}$ & 1970 & 1.12 & 0.04 & 2.18 & 1.29 & & & & \\
\hline \multirow[b]{2}{*}{ SB-VI } & & & & & 1.94 & 2.32 & 3.06 & 3.66 & 4.44 & 4.86 & & \\
\hline & C & $08^{\circ} 44.84^{\prime}$ & $119^{\circ} 16.09^{\prime}$ & 2000 & 0.76 & 0.15 & 0.85 & 1.59 & 0.94 & & & \\
\hline \multirow[b]{2}{*}{ SB-VII } & & & & & 2.00 & 2.32 & 2.68 & 3.16 & 3.58 & 3.74 & & \\
\hline & $\mathrm{C}$ & $08^{\circ} 25.59$ & $118^{\circ} 15.80^{\prime}$ & 810 & 0.49 & 0.93 & 0.82 & 0.51 & 1.13 & & & \\
\hline \multirow[b]{2}{*}{ SB-VIII } & & & & & 1.96 & 2.38 & 3.00 & & & & & \\
\hline & C & $08^{\circ} 22.40^{\prime}$ & $118^{\circ} 34.16^{\prime}$ & 1590 & 0.92 & 1.43 & & & & & & \\
\hline \multirow{3}{*}{ SB-IX } & & & & & 2.37 & 2.87 & 3.68 & 4.63 & 5.19 & & & \\
\hline & C & $08^{\circ} 55.23^{\prime}$ & $119^{\circ} 24.92^{\prime}$ & 1970 & 1.65 & 0.12 & 1.67 & 2.74 & & & & \\
\hline & & & & & 2.39 & 2.83 & 3.22 & 3.81 & 4.44 & & & \\
\hline SB-XII & C & $09^{\circ} 03.82^{\prime}$ & $119^{\circ} 23.72^{\prime}$ & 1920 & 2.04 & 0.17 & 0.43 & 1.91 & & & & \\
\hline SB-XIII & C & $09^{\circ} 21.32^{\prime}$ & $119^{\circ} 15.96^{\prime}$ & 2000 & $\begin{array}{l}1.74 \\
0.91\end{array}$ & $\begin{array}{l}1.83 \\
0.09\end{array}$ & $\begin{array}{l}2.15 \\
0.59\end{array}$ & 2.38 & & & & \\
\hline & & & & & 1.60 & 2.10 & 2.90 & 3.25 & 4.55 & & & \\
\hline SB-20 & D & $08^{\circ} 59.49^{\prime}$ & $119^{\circ} 20.62^{\prime}$ & 2000 & 0.64 & 0.53 & 0.70 & 2.26 & & & & \\
\hline & & & & & 1.55 & 2.30 & 2.50 & 2.70 & & & & \\
\hline SB-22 & D & $09^{\circ} 28.72^{\prime}$ & $119^{\circ} 58.98^{\prime}$ & 1340 & 0.57 & 0.59 & 0.75 & & & & & \\
\hline & & & & & $\begin{array}{l}1.58 \\
0.56\end{array}$ & 1.80 & $\begin{array}{l}2.30 \\
0.63\end{array}$ & 2.80 & 3.80 & & & \\
\hline SB-23 & D & $09^{\circ} 47.02^{\prime}$ & $120^{\circ} 27.20^{\prime}$ & 1540 & 0.56 & 0.12 & 0.63 & 1.11 & & & & \\
\hline
\end{tabular}

Note: $\mathrm{V}=$ seismic velocity in $\mathrm{km} / \mathrm{s}\left(\mathrm{V}^{*}=\right.$ assumed velocity), and $\mathrm{h}=$ thickness in kilometers, of the isovelocity layers derived from the seismic measurements.

Sources: A = Murauchi et al., 1973; B = Ludwig et al., 1979; C = Sonne cruise SO-23, H. S. Schröder, unpubl. data; and $\mathrm{D}=$ Valdivia cruise VA-16, H. Schröder, unpubl. data. 
Table 5. Refraction seismic and sonobuoy measurements in the southeast Sulu Sea.

\begin{tabular}{|c|c|c|c|c|c|c|c|c|c|c|c|c|}
\hline \multirow{2}{*}{$\begin{array}{l}\text { Name } \\
\text { of } \\
\text { station }\end{array}$} & \multirow[b]{2}{*}{ Source } & \multicolumn{2}{|c|}{ Location } & \multirow{2}{*}{$\begin{array}{l}\text { Water } \\
\text { depth } \\
\text { (m) }\end{array}$} & \multirow{2}{*}{$\begin{array}{l}\mathrm{V}_{1} \\
\mathrm{~h}_{1}\end{array}$} & \multirow{2}{*}{$\begin{array}{l}\mathrm{v}_{2} \\
\mathrm{~h}_{2}\end{array}$} & \multirow{2}{*}{$\begin{array}{l}V_{3} \\
h_{3}\end{array}$} & \multirow{2}{*}{$\begin{array}{l}\mathrm{v}_{4} \\
\mathrm{~h}_{4}\end{array}$} & \multirow{2}{*}{$\begin{array}{l}\mathrm{V}_{5} \\
\mathrm{~h}_{5}\end{array}$} & \multirow{2}{*}{$\begin{array}{l}\mathrm{V}_{6} \\
\mathrm{~h}_{6}\end{array}$} & \multirow{2}{*}{$\begin{array}{l}\mathrm{v}_{7} \\
\mathrm{~h}_{7}\end{array}$} & \multirow{2}{*}{$\begin{array}{l}\mathrm{v}_{8} \\
\mathrm{~h}_{8}\end{array}$} \\
\hline & & $\mathrm{N}$ & E & & & & & & & & & \\
\hline \multirow{3}{*}{ Profile 16} & & & & & $2.00^{*}$ & $3.4^{*}$ & 6.41 & 8.28 & & & & \\
\hline & A & $07^{\circ} 48^{\prime}$ & $120^{\circ} 04^{\prime}$ & 3900 & 0.53 & 2.24 & 4.01 & & & & & \\
\hline & & $07^{\circ} 21^{\prime}$ & $120^{\circ} 30^{\prime}$ & 4270 & 1.10 & 2.04 & 3.86 & & & & & \\
\hline \multirow{3}{*}{ Profile 17} & & & & & $2.00^{*}$ & 3.51 & 5.18 & 6.16 & 7.19 & & & \\
\hline & A & $06^{\circ} 43^{\prime}$ & $121^{\circ} 17^{\prime}$ & 50 & 0.05 & 1.34 & 2.92 & 5.02 & & & & \\
\hline & & $06^{\circ} 18^{\prime}$ & $121^{\circ} 38^{\prime}$ & 50 & 0.11 & 1.81 & 1.85 & 5.02 & & & & \\
\hline \multirow[b]{2}{*}{$309 \mathrm{C} 12$} & & & & & 2.05 & 2.45 & 2.75 & 3.10 & 3.50 & 3.80 & & \\
\hline & B & $06^{\circ} 27.9^{\prime}$ & $118^{\circ} 52.8^{\prime}$ & 620 & 1.10 & 0.41 & 0.61 & 0.39 & 0.65 & & & \\
\hline & & & & & 1.90 & 2.05 & 2.85 & & & & & \\
\hline $310 \mathrm{C} 12$ & B & $05^{\circ} 59.8^{\prime}$ & $119^{\circ} 20.2^{\prime}$ & 980 & 0.79 & 1.12 & & & & & & \\
\hline \multirow[b]{2}{*}{$41 \mathrm{C} 14$} & & & & & $1.67^{*}$ & $2.35^{*}$ & 4.45 & & & & & \\
\hline & B & $05^{\circ} 38.5$ & $119^{\circ} 48.8$ & 470 & 0.73 & 1.73 & & & & & & \\
\hline \multirow[b]{2}{*}{$42 \mathrm{C} 14$} & & & & & $1.8^{*}$ & 2.65 & & & & & & \\
\hline & B & $05^{\circ} 49.8^{*}$ & $119^{\circ} 53.6^{\prime}$ & 540 & 1.10 & & & & & & & \\
\hline \multirow[b]{2}{*}{$215 \mathrm{~V} 28$} & & & & & $1.74^{*}$ & $2.28^{*}$ & $3.14^{*}$ & 4.60 & & & & \\
\hline & B & $07^{\circ} 54.2^{\prime}$ & $120^{\circ} 06.7^{\prime}$ & 4040 & 0.43 & 0.48 & 0.89 & & & & & \\
\hline \multirow{3}{*}{ SB X } & & & & & 1.75 & 2.05 & 2.20 & 2.55 & 2.75 & 4.90 & 7.50 & \\
\hline & C & $06^{\circ} 12.33^{\prime}$ & $119^{\circ} 08.47^{\prime}$ & 670 & 0.50 & 0.21 & 0.77 & 1.01 & 3.67 & 3.25 & & \\
\hline & & & & & 1.83 & 3.00 & 3.94 & 4.67 & 5.50 & & & \\
\hline SB XI & C & $06^{\circ} 21.57^{\prime}$ & $119^{\circ} 25.87^{\prime}$ & 2950 & 0.92 & 1.34 & 0.82 & 0.64 & & & & \\
\hline
\end{tabular}

Note: $\mathrm{V}=$ seismic velocity in $\mathrm{km} / \mathrm{s}\left(\mathrm{V}^{*}=\right.$ assumed velocity $)$, and $\mathrm{h}=$ thickness in kilometers of the isovelocity layers derived from the seismic measurements.

Sources: A = Murauchi et al., 1973; B = Ludwig et al., 1979; and C = Sonne cruise SO-23, H. Schröder, unpubl. data.

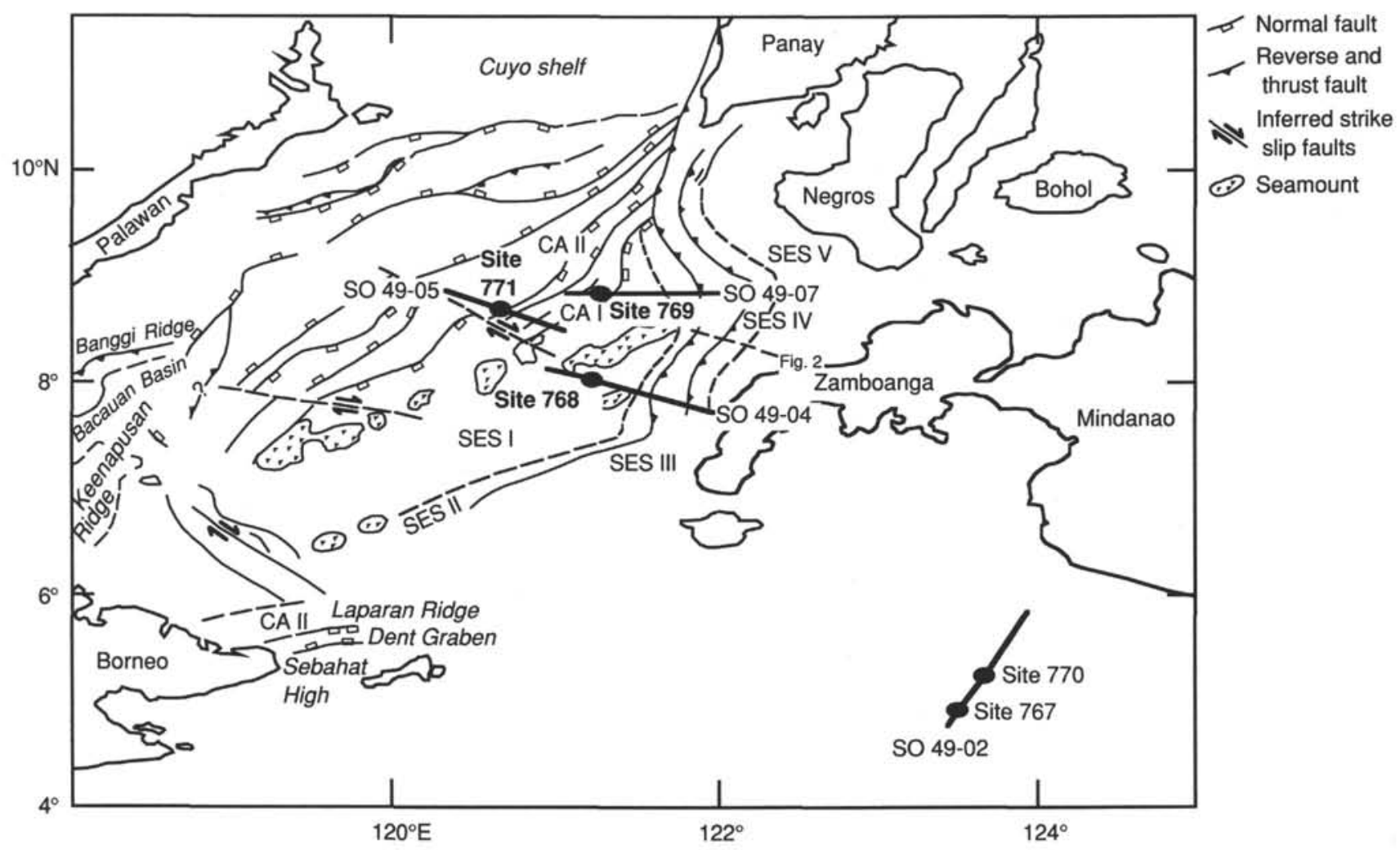

Figure 1. Structural map of the southeast Sulu Sea based on seismic line interpretation. 


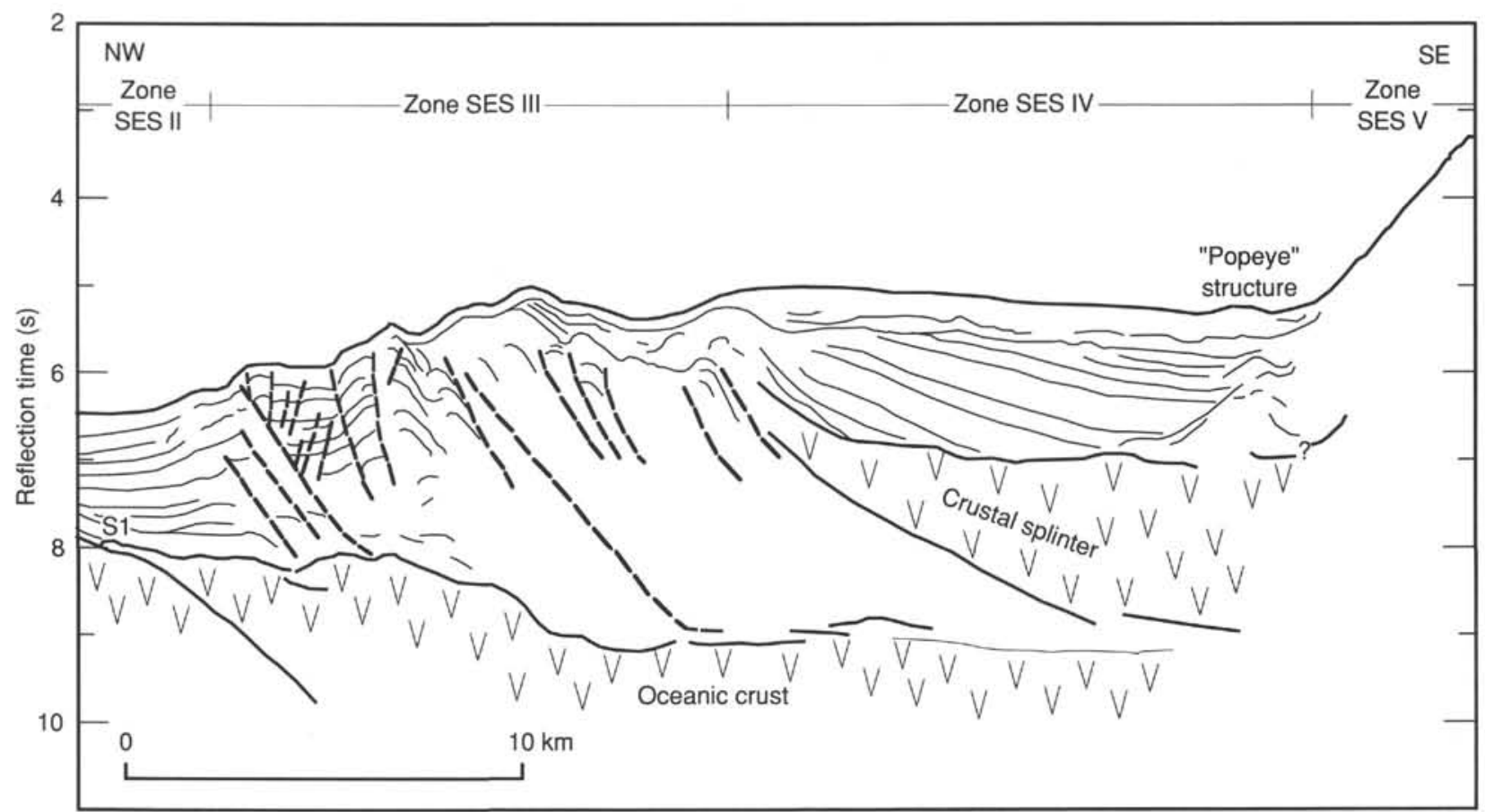

Figure 3. Interpretation of Line SO49-06 (SP-255-1000) showing presence of a crustal splinter in the Sulu Sea Trench inner wall (location on Fig. 1).

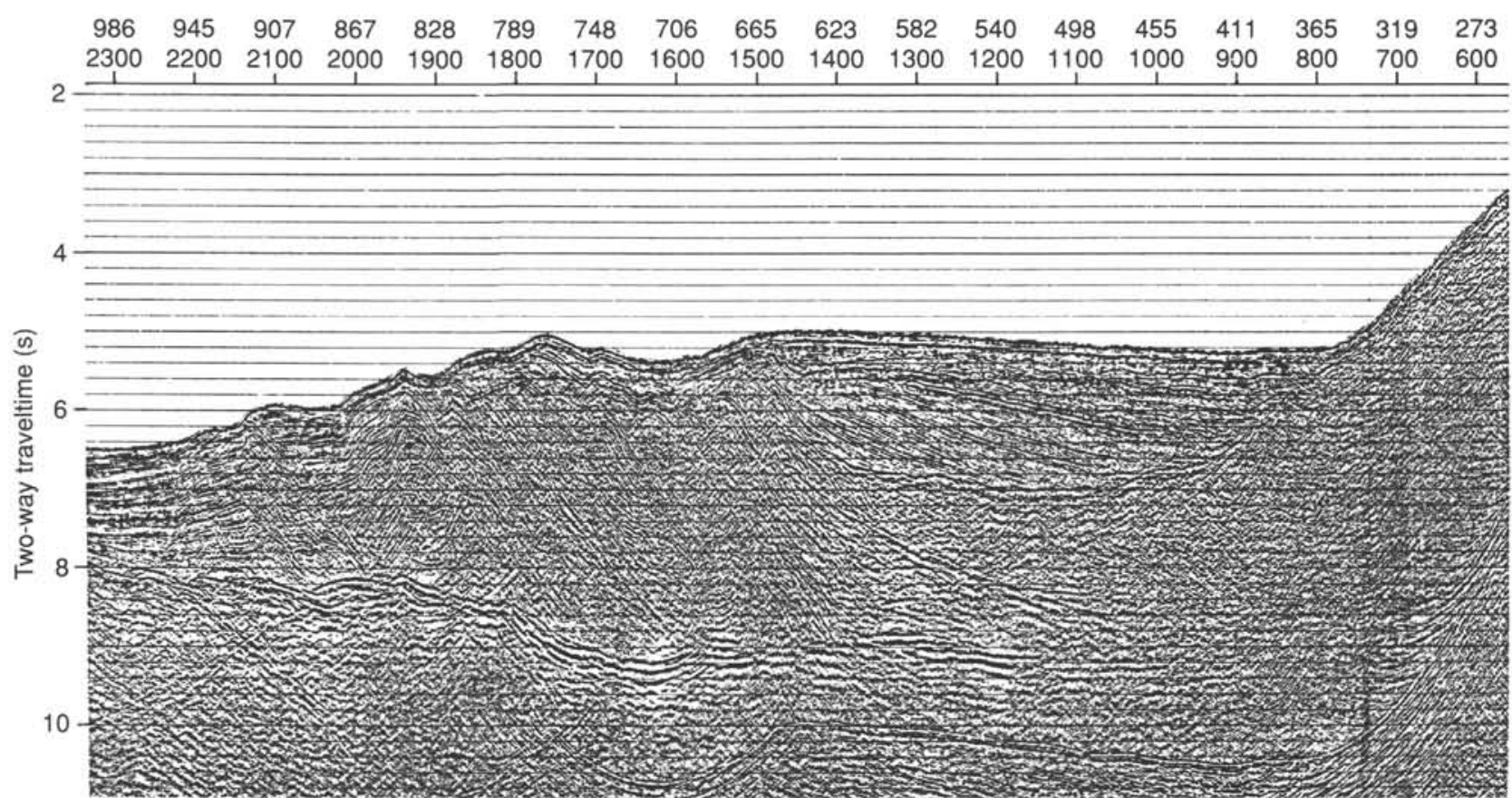

Figure 4. Seismic record of Line SO49-06 (SP-255-1000). Location on Figure 1. 